\title{
Genetic variability of human papillomavirus type 39 based on E6, E7 and L1 genes in Southwest China
}

Jiaoyu He ${ }^{1,2 \dagger}$, Tianjun $\mathrm{Li}^{1,2 \dagger}$, Youliang Wang ${ }^{3}$, Zhilin Song ${ }^{1,2}$, Qiufu Li ${ }^{1,2}$, Yiran Liu ${ }^{1,2}$, Yanru Cui ${ }^{1,2}$, Siyu Ma Ma, Junhang Deng ${ }^{1,2}$, Xia Wei ${ }^{1,2}$ and Xianping Ding ${ }^{1,2,4^{*}}$ (D)

\begin{abstract}
Background: Human papillomavirus type 39 associated with genital intraepithelial neoplasia and invasive cancers, has a high prevalence in Southwest China. HPV E6, E7 are two main papillomavirus oncoproteins, closely relate to the function of HPV immortalization, cell transformation, and carcinogenesis. L1 is the major capsid protein, can reflect the replication status of the virus in cells and the progression of cervical lesions. The purpose of this study is to reveal the prevalence of HPV 39 and the genetic polymorphisms of HPV39 based on E6, E7 and L1 gene in southwest China.

Methods: Cell samples were collected by cervical scraped for HPV detecting and typing, and HPV39 positive samples were selected out. Important E6, E7 and L1 genes of HPV39 were sequenced and analyzed for the study of HPV39 genetic polymorphisms. Phylogenetic trees were constructed by Maximum-likelihood and Kimura 2-parameters methods in Molecular Evolutionary Genetics Analysis version 6.0. The selection pressures of E6, E7 and L1 genes were estimated by Datamonkey web server. The secondary and three-dimensional structure of HPV39 E6, E7 proteins were created by sopma server and SWISS-MODEL software.
\end{abstract}

Results: 344 HPV39 positive samples were selected from 5718 HPV positive cell samples. Among HPV39 E6-E7 sequences, 20 single nucleotide mutations were detected, including 10 non-synonymous and 10 synonymous mutations; 26 single nucleotide mutations were detected in HPV39 L1 sequences, including 7 non-synonymous and 19 synonymous mutations respectively. 11 novel variants of HPV39 E6-E7 (5 in E6 and 6 in E7) and 14 novel variants of HPV39 L1 were identified in this study. A-branch was the most frequent HPV39 lineage in southwest China during our investigation. Selective pressure analysis showed that codon sites 26, 87, 151 in E6 and 75, 180, 222, 272, 284, 346, 356 in $L 1$ were positively selected sites, as well as codon sites $45,138,309,381$ were negative selection sites in $L 1$ gene, E7 has neither positive selection sites nor negative selection sites. A certain degree of secondary and three-dimensional structure dislocation was existed due to the non-synonymous mutations.

Conclusions: Amino acid substitution affected the secondary and three-dimensional structure of HPV39, and resulting in the differences of carcinogenic potential and biological functions as well as the immune response due to the antigen epitopes difference, the antigen epitopes with stronger adaptability in Southwest will be screened out based

\footnotetext{
*Correspondence: brainding@scu.edu.cn

†Jiaoyu He and Tianjun Li contributed equally to this work

${ }^{4}$ Institute of Medical Genetics, College of Life Sciences, Sichuan University, Chengdu 610064, China

Full list of author information is available at the end of the article
}

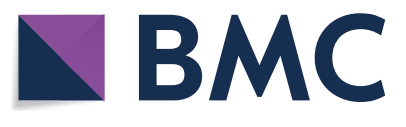

(c) The Author(s) 2021. Open Access This article is licensed under a Creative Commons Attribution 4.0 International License, which permits use, sharing, adaptation, distribution and reproduction in any medium or format, as long as you give appropriate credit to the original author(s) and the source, provide a link to the Creative Commons licence, and indicate if changes were made. The images or other third party material in this article are included in the article's Creative Commons licence, unless indicated otherwise in a credit line to the material. If material is not included in the article's Creative Commons licence and your intended use is not permitted by statutory regulation or exceeds the permitted use, you will need to obtain permission directly from the copyright holder. To view a copy of this licence, visit http://creativecommons.org/licenses/by/4.0/. The Creative Commons Public Domain Dedication waiver (http://creativeco mmons.org/publicdomain/zero/1.0/) applies to the data made available in this article, unless otherwise stated in a credit line to the data. 
on the above research results for the later vaccine development. And gene polymorphism of HPV39 in Southwest China may improve the effectiveness of clinical test and vaccine design, specifically for women in Southwest China.

Keywords: HPV39, Genetic variability, E6, E7 and L1 genes, Cervical cancer, Southwest china, Lineage phylogeny, protein structure

\section{Introduction}

Cervical cancer (CC) is the fourth leading cancer in women worldwide. The incidence of $\mathrm{CC}$ in developing countries is almost as twice as in developed countries, $17.8 \%$ and $9.0 \%$ respectively [1]. According to the report by the International Agency for Research on Cancer in 2018, the CC infection rate among Chinese women is 10.7 per 100,000 , just below the global rate of 13.1 per 100,000 , and increasing, that due to the neglect of screening [2]. Obviously, this cancer has seriously threatened the life and health of Chinese women.

HPV is a small, non-enveloped and double stranded DNA (dsDNA) virus, approximately 8,000 base pairs (bp) which contains early region (E6, E7, E1, E2, E4, and E5) and late region (L1, L2) [3]. High-risk human papilloma virus (HR-HPV) persistent infection is the leading cause of CC. E6 and E7 can inhibit the tumor suppressor pathways of $\mathrm{p} 53$ and $\mathrm{pRb}$ (retinoblastoma) respectively, that resulting in immortalization, cell transformation, and carcinogenesis, are two main papillomavirus oncoproteins [4-7]. L1 is the major capsid protein, which can reflect the replication status of virus in cells, the detection of L1 capsid protein expression can predict the progression and degeneration of cervical lesions [8].

More than $200 \mathrm{HPV}$ types had been recognized by the International HPV Reference Center [9]. A large number of epidemiological and laboratory research data show that $12 \mathrm{~h}-\mathrm{HPV}$ are closely related to the occurrence of malignant lesions, such as cervical cancer, including HPV16, 18, 31, 33, 35, 39, 45, 51, 52, 56, 58, and 59, and they mainly belong to the $\alpha-9$ (HPV16-related) and $\alpha-7$ (HPV18-related) genus based on the principle of evolutionary classification $[10,11]$.

Globally, HPV16 and HPV18 are the most common HR-HPV, persistence infection associated with those causing $70 \%$ of $\mathrm{CC}$ and pre-cancerous cervical lesions [12]. HPV39 belongs to $\alpha-7$, has no available commercial vaccines, due to its low infection rate [13]. Nevertheless, the infection rate of different HPV genotypes varies in different geographical regions and different ages [1]. For example, in southern China, HPV infections occurs mainly in women under 30 years old, and HPV52, HPV16, HPV58, HPV18 and HPV39 are the most common HR-HPV subtypes [14]. In western China, HPV16, HPV52, HPV58, HPV33 and HPV18 are the most prevalence HR-HPV subtypes among patients with cervical intraepithelial neoplasia (CIN), HPV39 ranking eighth [15]. In our research, the detection rate of HPV39 is higher than that of HPV18 (Fig. 1). Nowadays, HPV18 has been extensively studied. While, the data available on HPV39 is still limited, especially in China. Therefore, the genetic polymorphisms, lineage phylogeny and positive selections of HPV39 based on $E 6, E 7$ and $L 1$ genes in southwest China from a large amount of samples are sorely needed for detection of HPV-related diseases and development of HPV vaccine, as well as increased the attention to the China CC related $\alpha-7$ HPV (HPV18, 39, 45, 59, 68, 70, 85 and 97).

\section{Materials and methods \\ Samples resource}

23,054 specimens were randomly collected from January 2015 to August 2019 in the Sichuan Reproductive Health Research Center Affiliated Hospital, Chengdu Song zi niao Sterility Hospital, Infertility Hospital Affiliated to Chengdu Medical College, Chengdu Jinsha hospital, and Angel Women's and Children's Hospital. The cervical swabs were placed in $-20^{\circ} \mathrm{C}$ antiseptic buffer for HPV detection and typing. The study was ethically approved by the Education and Research Committee and Ethics Committee of Sichuan University, Sichuan, China. All data was used with the consent of HPVtested patients.

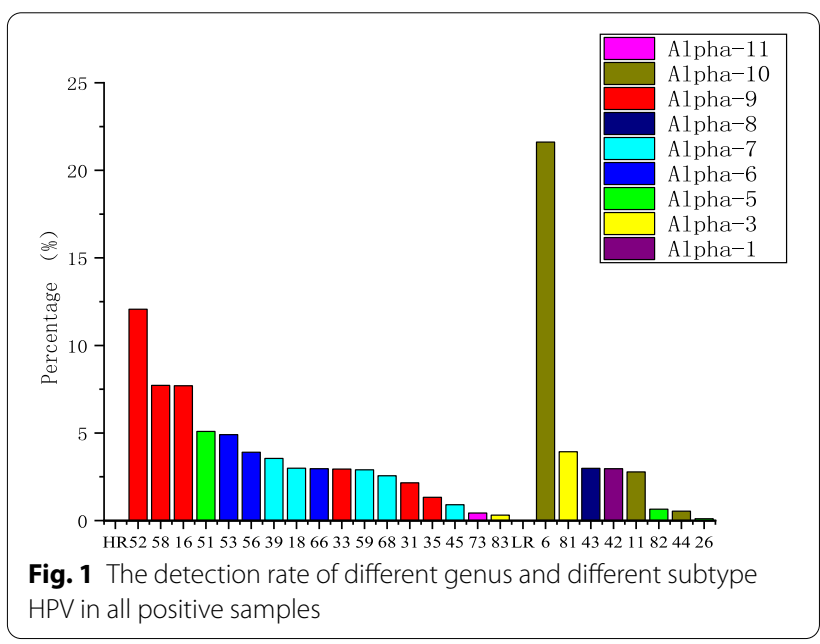




\section{Genomic DNA extraction and HPV typing}

The viral genomic DNA was extracted by the HEALTH Nucleic Acid Extraction Kit (Ningbo, Zhejiang) and genotyped by 3500DX Genetic Analyzer (Ningbo, Zhejiang), according to the manufacturer's instructions. The gene analyzer can classify $17 \mathrm{~h}$ /probable HR-HPV types (16, $18,31,33,35,39,45,51,52,53,56,58,59,66,68,73,83$ ) and 8 LR-HPV types $(6,11,26,42,43,44,81$ and 82$)$ based on capillary electrophoresis. 3,087 specimens belonging to $\alpha-7(18,39,45,59,68)$ and $\alpha-9(16,31,33,35,52,58)$ were detected, of which 344 were HPV39. The extracted DNA was saved at $-20^{\circ} \mathrm{C}$ for follow-up experiments.

\section{PCR amplification and Variant identification}

The primers of HPV39 E6, E7 and $L 1$ were designed by PRIMER 5.0 based on the HPV39 reference sequences (GeneBank number: M62849) (Table 1) and synthesized by TSINGKE (Chengdu, China). $5 \mu$ HPV39 DNA, $13.1 \mu \mathrm{l} \mathrm{ddH_{2 }} \mathrm{O}, 1 \mu \mathrm{l}$ primers, $0.4 \mu \mathrm{l}$ transtaq DNA polymerase, $2.5 \mu \mathrm{l}$ dNTPS, and $3 \mu \mathrm{l}$ buffer form the PCR reaction system. PCR conditions were: $95{ }^{\circ} \mathrm{C}$ for $6 \mathrm{~min}, 34$ cycles according to the following protocol: denaturation at $94{ }^{\circ} \mathrm{C}$ for $45 \mathrm{~s}$, annealing at $57^{\circ} \mathrm{C}$ for $E 6, E 7$ and $L 1$ for $50 \mathrm{~s}$ and elongation at $72{ }^{\circ} \mathrm{C}$ for $60 \mathrm{~s}$. The PCR products were visualized by gel electrophoresis in $2 \%$ agarose gel (Sangon Biotech Co., Ltd.). The target products of E6, $E 7$ and $L 1$ were purified and sequenced by TSINGKE (Chengdu, China). The complete alignment results of the successfully amplified 306 E6-E7 and 298 L1 sequences were all arranged in Tables 2 and 3 respectively.

\section{Sequence analysis}

Genetic polymorphisms and phylogenetic analysis of HPV39 E6/E7/L1 genes

The E6, $E 7$ and $L 1$ sequences obtained in this study were aligned and compared to M62849 by MEGA 6 software. Nucleotide sequences were converted to amino acid sequence by MEGA 6 , in order to determine the amino acid changes caused by nucleotide changes. Maximumlikelihood trees for HPV39 isolates were constructed by 807 bp E6-E7 and 1518 bp $L 1$ nucleotide sequences respectively with Kimura's two-parameter model in MEGA 6. The data was bootstrap resampled 500 times for tree topology evaluation.

\section{Selective pressure analysis of HPV39 E6/E7/L1 genes}

The selection pressure of HPV39 E6, E7 and $L 1$ were predicted by Datamokey online analysis software [16, 17]. The normalized posterior mean of the $\mathrm{dN}-\mathrm{dS}$ difference and the Bayesian posterior probability $(\mathrm{dN}>\mathrm{dS})$ of the positive selection for each codon position were obtained. A Bayes factor greater than 50 indicates that a site is positively selected.

\section{The secondary and three-dimensional structure of HPV39 E6/ E7 protein}

The secondary structure of main carcinogenic protein HPV E6/E7 reference sequence and the amino acid sequence with mutation were predicted by sopma server (self-optimized prediction method) [18], to analyze whether the non-synonymous mutation cause changes in the protein secondary structure or not. Sopma is a server that uses five independent methods (Levin homologous prediction method, gor method, dual prediction method, $\mathrm{PhD}$ method and sopma method) to predict and synthesize a "consistent prediction result".

The three-dimensional spatial structure formed by the interaction between secondary structure. The three-dimensional structure of HPV39 E6/E7 reference and mutation sequence were constructed from scratch to understand the effect of non-synonymous mutation. I-tasser (iterative thread optimization) [19] is a hierarchical method for protein structure and function prediction, it identifies the structure template from PDB by means of lomets, and constructs the full-length atom model by segment assembly simulation based on

Table 1 The primers of HPV39 E6/E7/L1

\begin{tabular}{|c|c|c|c|c|}
\hline Gene & Primer sequences & Primer location (nt) & Amplification length (bp) & $\begin{array}{l}\text { Annealing } \\
\text { temperature } \\
\left({ }^{\circ} \mathrm{C}\right)\end{array}$ \\
\hline \multirow[t]{2}{*}{ E6 } & F: 5'-GGGAGTAACCGAAAACGGTC-3' & $36-55$ & 821 & 57 \\
\hline & R: 5'-GTTGTCGCAGAGTATCCCGT-3' & $856-837$ & & \\
\hline \multirow[t]{2}{*}{ E7 } & F: 5'-GGACCGCAGACTAACACGAA-3' & $187-206$ & 613 & 57 \\
\hline & R: 5'-CCCTTTGGGCCTCTTGCATA-3' & $799-780$ & & \\
\hline \multirow[t]{2}{*}{ L1-1 } & F: 5'-GCTCACTACCTTCTGTGGCTT-3' & $105-125$ & 1059 & 57 \\
\hline & R: 5'-ACCCACCATACCACCACGAT-3' & $1163-1144$ & & \\
\hline \multirow[t]{2}{*}{$41-2$} & F: 5'-ATTGGGGAGCACTGGGGTAA-3' & $72-91$ & 1332 & 56 \\
\hline & R: 5'-ACTTCGGTCGCCACAAAATG-3' & $1403-1384$ & & \\
\hline
\end{tabular}




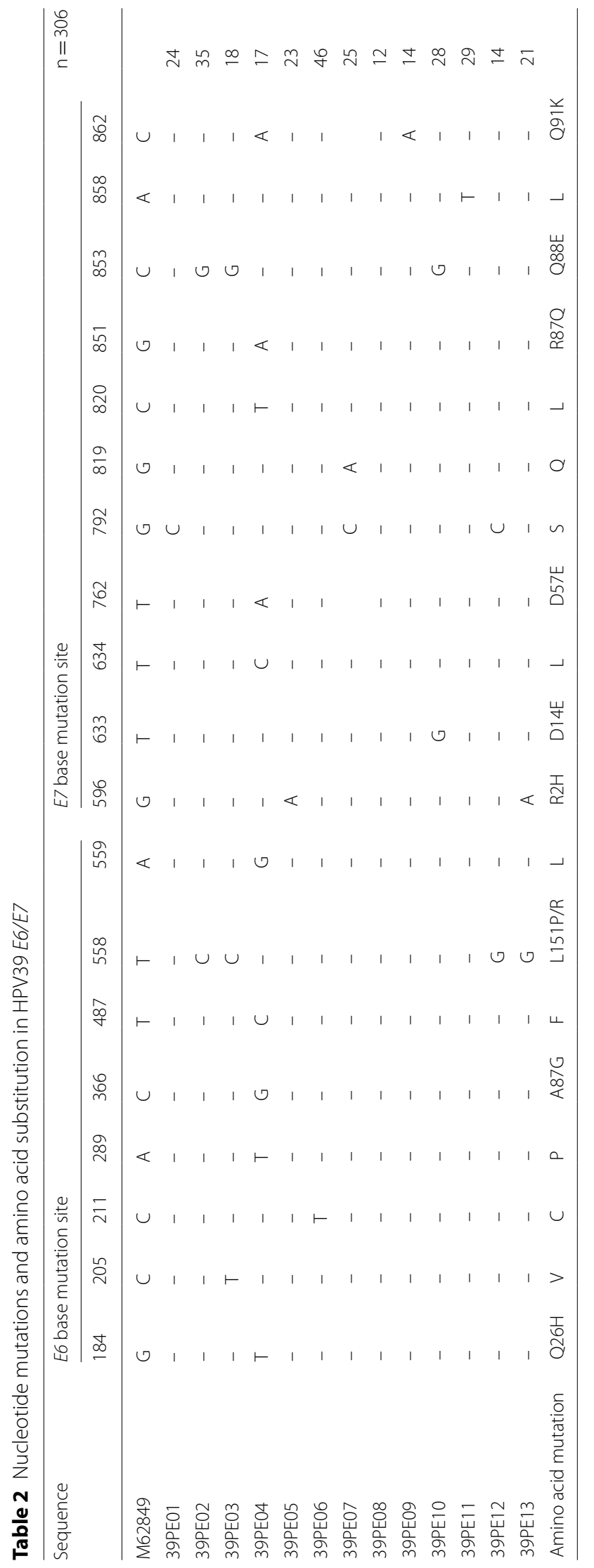




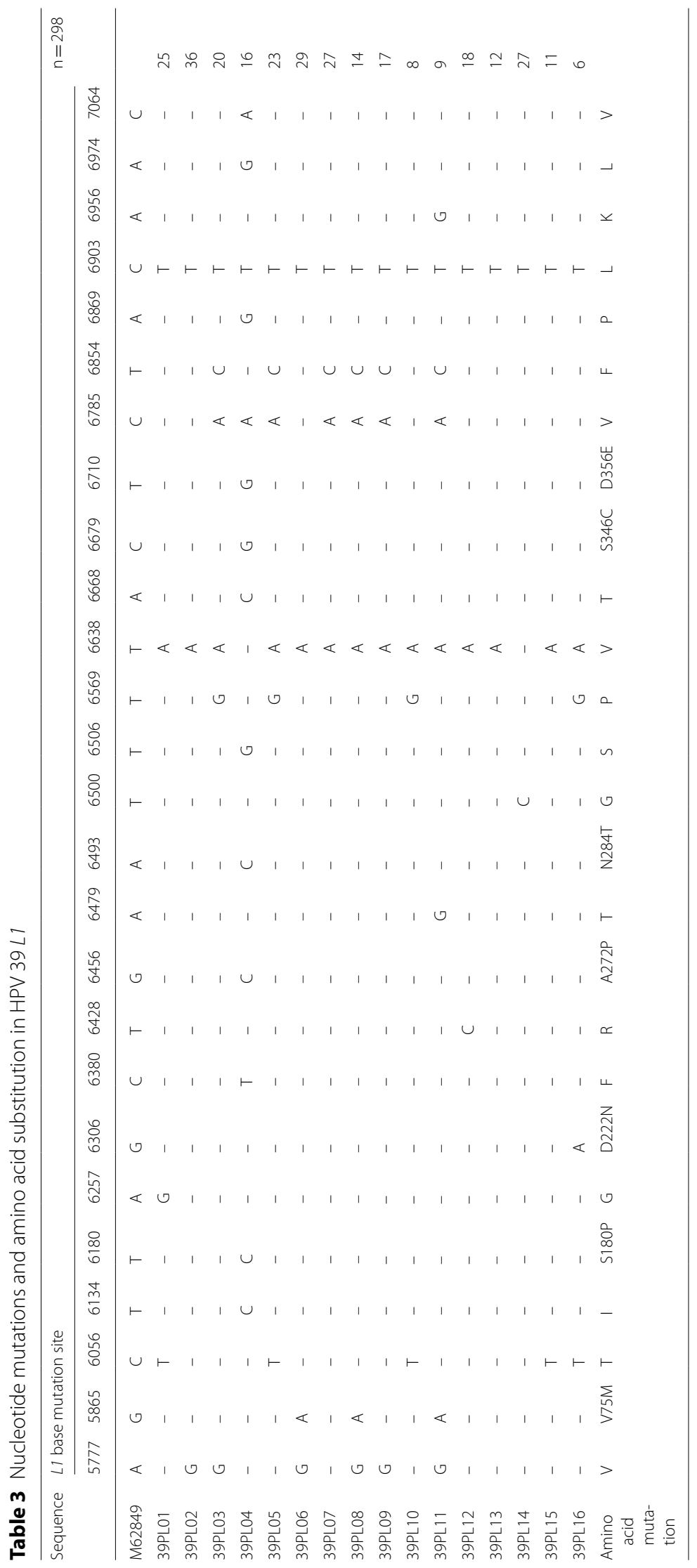


iterative template, then, the three-dimensional model is reprogrammed into thread through protein functional database biolip to get the target protein sequence, thirdly optimized prediction model and minimized energy by Swiss-Pdb Viewer [20] to evaluate and verify the accuracy of HPV39 E6/E7 reference and mutation sequence generation model. The model was visualized, and the three-dimensional structure of reference and mutation sequence were compared to mark the amino acid mutation site for analyzing whether the gene mutation affected on the three-dimensional structure of the protein or not by PyMOL 2.2.0 [21].

\section{Results}

\section{The prevalence of HPV39 in Southwest China}

344 HPV39 cases were detected in all 23,054 samples, including 334 women and 10 men, accounting for $1.49 \%$ of the total samples, and $6.02 \%(344 / 5718)$ of HPV positive samples, ranking seventh in HR-HPV detection rate and first in $\alpha-7 \mathrm{~h}-\mathrm{HPV}$ among all positive samples (Fig. 1). The detection rates of HPV39 in all positive samples during recent five years were changed, $4 \%(39 / 973)$ in $2015,8.13 \%(67 / 824)$ in $2016,4.05 \%$ $(71 / 1752)$ in $2017,6.20 \%(91 / 1468)$ in 2018 and $10.84 \%$ (76/701) in 2019 (Fig. 2). In 344 HPV39 positive samples, 158 cases (45.93\%) were single infection, 186 cases (54.07\%) were multiple infection. Among the HPV39 co-infection, mixed infection with HPV6 was the most common (18.49\%), followed by HPV16 (12.5\%), HPV73 and HPV83 had no mixed infection with HPV39 (Table 4). Age is an important determination of HPV infection risk. Among the HPV39 positive population, $18-28$ years old $(52.62 \%)$ was the highest detection rate age group. The age group of younger than 18 years old was the lowest with $1.16 \%$ detection rates (Table 5).

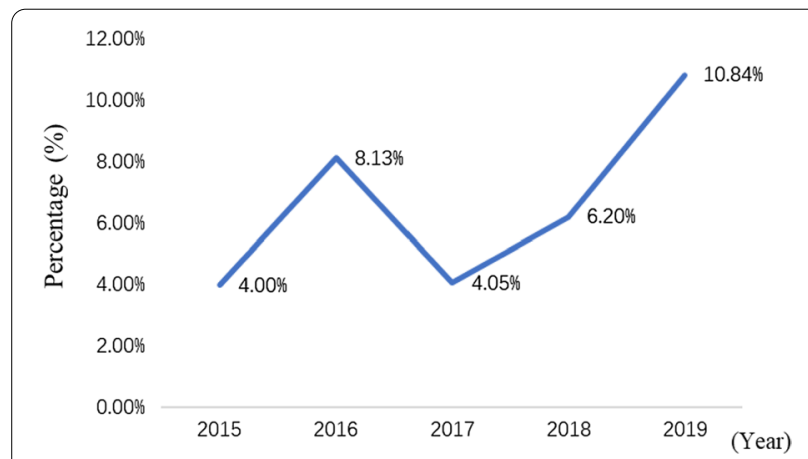

Fig. 2 The detection rate of HPV39 in all positive samples during 2015-2019
Table 4 The detection rates of HPV39 with other HPV types co-infection

\begin{tabular}{llllll}
\hline Type & Number & Percent (\%) & Type & Number & percent (\%) \\
\hline 6 & 71 & 18.49 & 33 & 8 & 2.08 \\
16 & 48 & 12.5 & 35 & 8 & 2.08 \\
52 & 43 & 11.2 & 53 & 8 & 2.08 \\
68 & 35 & 9.11 & 31 & 7 & 1.82 \\
58 & 32 & 8.33 & 59 & 7 & 1.82 \\
51 & 20 & 5.21 & 45 & 4 & 1.04 \\
81 & 20 & 5.21 & 43 & 4 & 1.04 \\
56 & 17 & 4.43 & 82 & 2 & 0.52 \\
42 & 16 & 4.17 & 44 & 1 & 0.26 \\
18 & 13 & 3.39 & 73 & 0 & 0 \\
66 & 13 & 3.39 & 83 & 0 & 0 \\
11 & 9 & 2.34 & & & \\
\hline
\end{tabular}

\section{Sequence analysis of HPV39 E6/E7/L 1 genes}

Among the 344 HPV39 positive specimens, 306 (88.95\%) E6-E7 and 298 (86.63\%) L1 sequences were amplified and analyzed successfully. All sequences obtained in this study have been uploaded to the GenBank with accession number as follows: E6-MK340878-MK340882; E7-MK340883-MK340890; L1-MK344658- MK344673.

Compared with the reference sequence M62849, 306 HPV39 E6-E7 sequences obtained in this study showed that $94.12 \%(288 / 306)$ sequences were variant. 19 single nucleotide mutations ( 8 in $E 6$ and 11 in $E 7$ ) were detected, the mutation rate of $E 6$ was $1.68 \%(8 / 477)$, and $E 7$ was $3.33 \%$ (11/330). Among them, 9 (9/19, 47.37\%) were non-synonymous mutations and $10(10 / 19,52.63 \%)$ were synonymous mutations. Moreover, the nucleotide mutations of C205T, C211T, A289T, T487C, T558G in E6 and T633G, G792C, G819A, G851A, A858T, C862A in $E 7$ have not been reported. In addition, two mutations were observed at 558 sites of $E 6$, namely T558C (39pe02, 39pe03) and T558G (39pe12, 39pe13) (Table 2).

Compared with the reference sequence M62849, 298 HPV39 L1 variant sequence were obtained in 298 HPV39

Table 5 The HPV detection rates in all age groups among HPV39 positive samples

\begin{tabular}{lcl}
\hline Age (year) & Number & Percent (\%) \\
\hline$<18$ & 4 & 1.16 \\
$18-28$ & 181 & 52.62 \\
$29-39$ & 100 & 29.70 \\
$40-50$ & 45 & 13.08 \\
$51-61$ & 7 & 2.03 \\
$>61$ & 7 & 2.03 \\
Total & 344 & 100 \\
\hline
\end{tabular}


L1 sequence (100\%, 298/298). 26 single nucleotide mutations were detected, $26.92 \%(7 / 26)$ were non-synonymous mutations and $73.08 \%(19 / 26$,) were synonymous. In $L 1$ sequence, C6056T, A6257G, G6306A, T6428C, G6456C, A6479G, T6500C, T6506G, T6569G, C6679G, A6869G, T6710G, A6956G and A6974G have not been reported before. C6903T exists in all obtained sequences, details were shown in Table 3.

\section{Phylogenetic analysis and selective pressure analysis of HPV39 E6-E7 and $L 1$ sequences}

The ML phylogenetic tree of 13 isolates HPV39 E6-E7 was constructed, which obtained in the study, denoted as 39PE01-39PE13, accompanied by 18 reference sequences. HPV39 variants complete genome sequences were clustered into A (A1 and A2) and B two lineages [20], in our study, only one lineage B variant was detected (Fig. 3). The number of HPV39 isolates sub-lineages A1, A2 and lineage B variants were 58 (28.16\%), 141 (68.54\%) and 7 (3.39\%), respectively. 39PE06 was the most common variant $(44.83 \%, 26 / 58)$ in A1 sub-lineage and 39PE02 was the most common variant $(17.02 \%, 24 / 141)$ in A2 sub-lineage.

The ML phylogenetic tree of 16 isolates HPV39 L1 was constructed, denoted as 39PL01-39PL16, accompanied by 20 reference sequences (Fig. 4). According to the previously described lineages A (A1 and A2) and B, the ML phylogenetic tree shows the dichotomy of branches [20]. In the A lineage, there were 15 variants, and B lineage was one only. The number of HPV39 L1 isolates sublineages A1, A2 and lineage B variants were 114 (57.58\%), $78(39.39 \%)$ and $6(3.03 \%)$, respectively. 39PL02 was the most common variant $(21.93 \%, 25 / 114)$ in A1 sub-lineage and 39PL05 was the most common variant (26.92\%, 21/78) in A2 sub-lineage.

10 positively selected codon sites and 4 negatively selected codon sites were identified (Tables 6 and 7). Concretely, codon sites 26, 87, 151 in E6 and 75, 180, 222, 272, 284, 346, 356 in L1 are positively selected sites. Codon sites 45, 138, 309, 381 are negative selection sites in the L1 gene.

\section{The secondary and three-dimensional structure of HPV39 E6/E7 protein}

Three E6 variants, 39pe02, 39pe04, 39pe12 and five E7 variants, 39pe02, 39pe04, 39pe05, 39pe09, 39pe10 were detected in our research. The secondary structure of E6 mutation sequence showed that Q26H, L51P and L151R appeared in random crimp $(\mathrm{C})$, and $\mathrm{A} 87 \mathrm{G}$ appeared in $\alpha$-helix (H). Compared with reference sequence M62849, the secondary structure of L151P, L151R and Q26H changed from $\mathrm{H}$ to $\mathrm{C}$, and the amino acids substitution also influenced the secondary structure around them
( $\mathrm{H}$ shortened but $\mathrm{C}$ increased), $\mathrm{A} 87 \mathrm{G}$ did not change the secondary structure. For HPV39 E7, R2H and D57E appeared in C, D14E, R87Q, Q88E and Q91K appeared in $\mathrm{H}$, and there was no difference with M62849 secondary structure (Figs. 5 and 6).

The three-dimensional structure comparison of E6, E7 reference and mutant protein were shown in Figs. 7 and 8. For E6, the comparison chart of M62849, 39PE02 and 39PE12, L151P/R located in the surface and c-terminal of E6 protein 3D structure, the 151th amino acid substitution may lead the protein more closely to the environment; The comparison chart of M62849 and 39PE04, A87G amino acid substation located in helix, as well as Q26H, they are all exposed to the environment and near to the zinc granule. For E7, the comparison chart of M62849 and HPV39PE02, Q88E located in helix and exposed; The protein structure of M62849 and 39PE04 are difference in $57,87,91$ position, D57E located in coil and exposed, R87Q and Q91K are both in helix and inside of the protein; The comparison chart of M62849 and HPV39PE10, Q91K is located inside of E7 protein; The comparison between M62849 and 39PE10, D14E located in helix and exposed, while Q88E located in the helix and buried. Non-synonymous mutations somewhat changed the trend of E6/E7 and carboxyl end structural disorder, especially E7.

\section{Discussion}

HR-HPV persistent infection closely related to the occurrence of cervical precancerous lesions and CC [22], its carcinogenicity is mainly realized by E6, E7 oncoproteins encoded by HPV E6, E7 genes, that lead to genetic instability and cell growth uncontrolled [23, 24]. L1 major capsid proteins can be used to guide the design of prophylactic vaccines and induce the synthesis of high levels neutralizing antibodies major components, virus-like particles (VLP) [25]. HPV prevalence in southwest China during 2014-2019 was studied, demonstrating the significance of HPV39. Considering the relatively high prevalence of HPV39 in southwest China, we selected HPV39 samples to explore the gene polymorphism of E6, E7 and L1.

In this study, 344 HPV39 positive specimens were detected, accounting for $6.01 \%$ of the total positive population, higher than that of in Beijing (2.72\%), ranking seventh in HR HPV infection rate and first in $\alpha-7$ HPV; In the past five years, the positive rate of HPV39 was significantly increased, except 2016-2017, all suggested that the prevalence of HPV39 is relatively high in Southwest China and the environment of Southwest China is conducive to the survival of HPV39, so more attention should be paid to the prevention and control of this subtype. The HPV39 multiple infection rate is higher than 


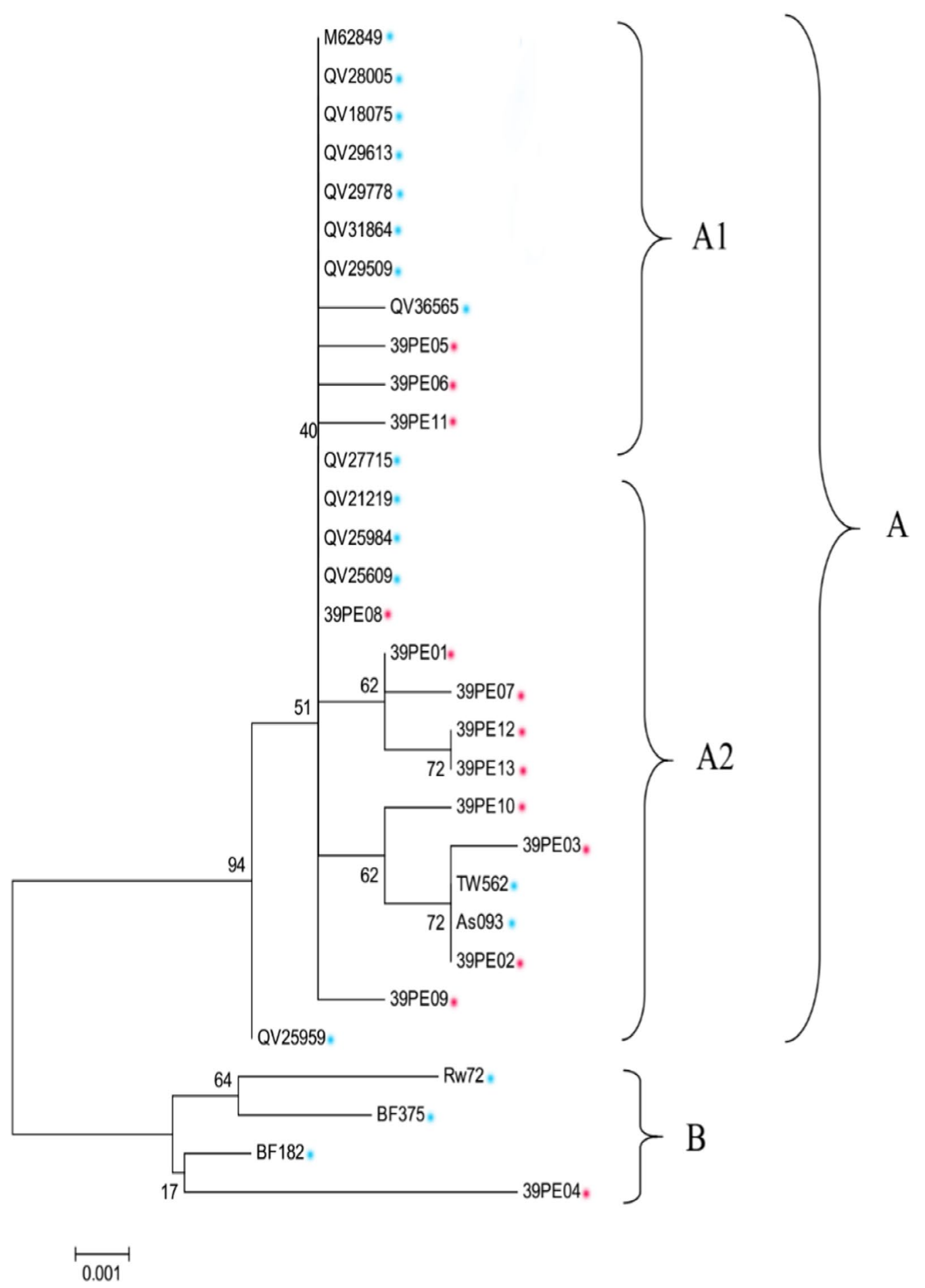

Fig. 3 The Maximum-likelihood trees of HPV39 E6-E7

that of in single infection, may due to the limitation of sample quantity, the different detection categories, and accuracy in different detection methods. Previous studies showed that HPV39 and HPV68 had the most mixed infection [26], but in our study, was HPV39 and HPV6 (18.49\%), the probability of mixed infection with HPV68 was $9.11 \%$, ranking fourth, above may due to HPV mixed infection prevalence in different regions was slightly 


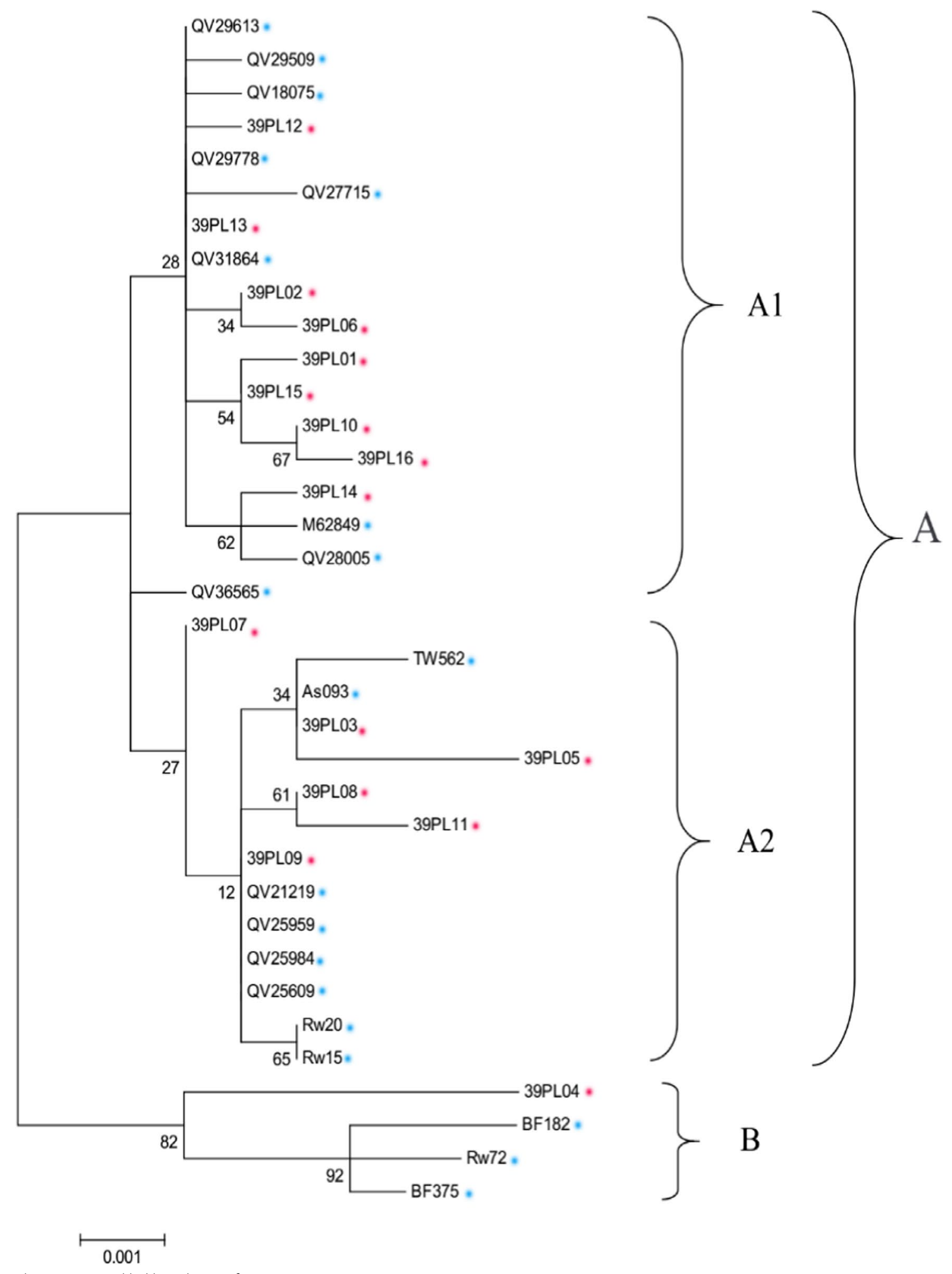

Fig. 4 The maximum-likelihood tree of HPV39 L1 
Table 6 HPV39 E6/E7/L1 positive selection site

\begin{tabular}{|c|c|c|c|c|c|c|}
\hline \multicolumn{7}{|c|}{ Positively selected sites (50 significance level) } \\
\hline Gene & Codon & $E[d S]$ & $E[d N]$ & Normalized E[dN-dS] & Posterior Probability & Bayes Factor \\
\hline & 26 & 1 & 10.0336 & 9.03359 & 0.929216 & 419.403 \\
\hline \multirow[t]{2}{*}{ E6 } & 87 & 1 & 10.0987 & 9.0987 & 0.935251 & 461.476 \\
\hline & 151 & 1 & 10.7965 & 9.79648 & 0.999941 & 538.503 \\
\hline \multirow[t]{4}{*}{ E7 } & \multicolumn{6}{|c|}{ Found no Positively selected sites (50 significance level) } \\
\hline & 75 & 0.561634 & 2.3459 & 1.78427 & 0.965567 & 587.849 \\
\hline & 180 & 0.626187 & 2.25204 & 1.62585 & 0.921948 & 247.618 \\
\hline & 222 & 0.7726 & 2.24721 & 1.47461 & 0.908844 & 209.008 \\
\hline \multirow[t]{4}{*}{ L1 } & 272 & 0.438175 & 2.25315 & 1.81497 & 0.936722 & 310.324 \\
\hline & 284 & 0.514167 & 2.25028 & 1.73612 & 0.929747 & 277.434 \\
\hline & 346 & 0.649058 & 2.25464 & 1.60559 & 0.921272 & 245.31 \\
\hline & 356 & 0.840996 & 2.24741 & 1.40641 & 0.903727 & 196.783 \\
\hline
\end{tabular}

Table 7 HPV39 E6/E7/L1 negative selection sites

\begin{tabular}{|c|c|c|c|c|c|c|}
\hline \multicolumn{7}{|c|}{ Negatively selected sites (50 significance level) } \\
\hline Gene & Codon & $E[d S]$ & $\mathrm{E}[\mathrm{dN}]$ & Normalized E[dN-dS] & Posterior Probability & Bayes Factor \\
\hline E6 & \multicolumn{6}{|c|}{ Found no negatively selected sites (50 significance level) } \\
\hline \multirow[t]{2}{*}{ E7 } & \multicolumn{6}{|c|}{ Found no negatively selected sites (50 significance level) } \\
\hline & 45 & 12.7368 & 0.088334 & -12.6485 & 0.999999 & $44,212.9$ \\
\hline \multirow[t]{3}{*}{ L1 } & 138 & 12.6934 & 0.088682 & -12.6048 & 0.999874 & 379.199 \\
\hline & 309 & 12.7055 & 0.088391 & -12.6172 & 0.999909 & 526.047 \\
\hline & 381 & 12.7033 & 0.088331 & -12.615 & 0.999903 & 491.748 \\
\hline
\end{tabular}

different. Age is an important HPV infection affect factor, previous study showed that $20-24$ and $50-54$ years old are the two HPV detection peaks age group. The first peak may due to the relatively active sexual lives of women in this age group. The change of female hormone secretion may result in the second positive peak, with the physiological and immune dysfunction, that leads to the resurrection of latent HPV infection and reduced ability to self-return after HPV infection [3]. In our study, HPV39 infection rate was the highest in 18-28 years old, consistent with the previous study results, indicating that age is an important HPV infection rate affecting factor. However, the second peak did not appear in Southwest China, that possibly because the above two infection peaks were the statistical analysis results of all HPV-positive patients, while this study only focuses on the statistical analysis of HPV39 positive patients, fewer age 50-54 participants participate in this study and the high age group is not the HPV39 susceptible population, resulting in the peak age of HPV39 positive patients aged $50-54$ years old lacking.

HPV E6, E7 and $L 1$ gene of 344 genital secretion samples were sequenced to analyze the gene variation, the distribution of HPV39 mutant and the effect of non-synonymous mutation on protein structure and function in this area. Our study showed that $E 7(3.33 \%)$ had the highest mutation rate, followed by $L 1(1.71 \%)$ and $E 6$ (1.68\%), indicating that $E 6$ may be a more suitable target for HPV therapeutic vaccine than $E 7$ and $L 1$; Inconsistent with previous research results that $E 7$ is a more suitable target for HPV therapeutic vaccines $[13,27]$, that may due to the target of HPV therapeutic vaccine is different in different HPV types and the limitation of sample size. In the HPV39 E6, $E 7$ and $L 1$ sequence, 29 (5 in E6, 8 in $E 7$ and 16 in $L 1$ ) mutation sequences were detected, comparing with reference sequence of $E 6$ (mk340878-mk340882), E7 (mk340883-mk340890) and L1(mk344658-mk344673). The maximum variation of $E 6, E 7$ and $L 1$ gene in the positive samples was $1.05 \%$ (5/477), 1.52\% (5/330) and 0.92\% $(14 / 1518)$ respectively, inconsistent with previously reported in other regions $(0.63 \%, 0.91 \%, 1.12 \%)$, suggesting that the variation in HPV39 E6, E7 and $L 1$ might be related to the distribution of regions and populations with regional characteristics.

HPV $39 E 6, E 7$ and $L 1$ gene belongs to A1, A2 and B lineages, and A pedigree outstand (94.45\% for E6-E7; 


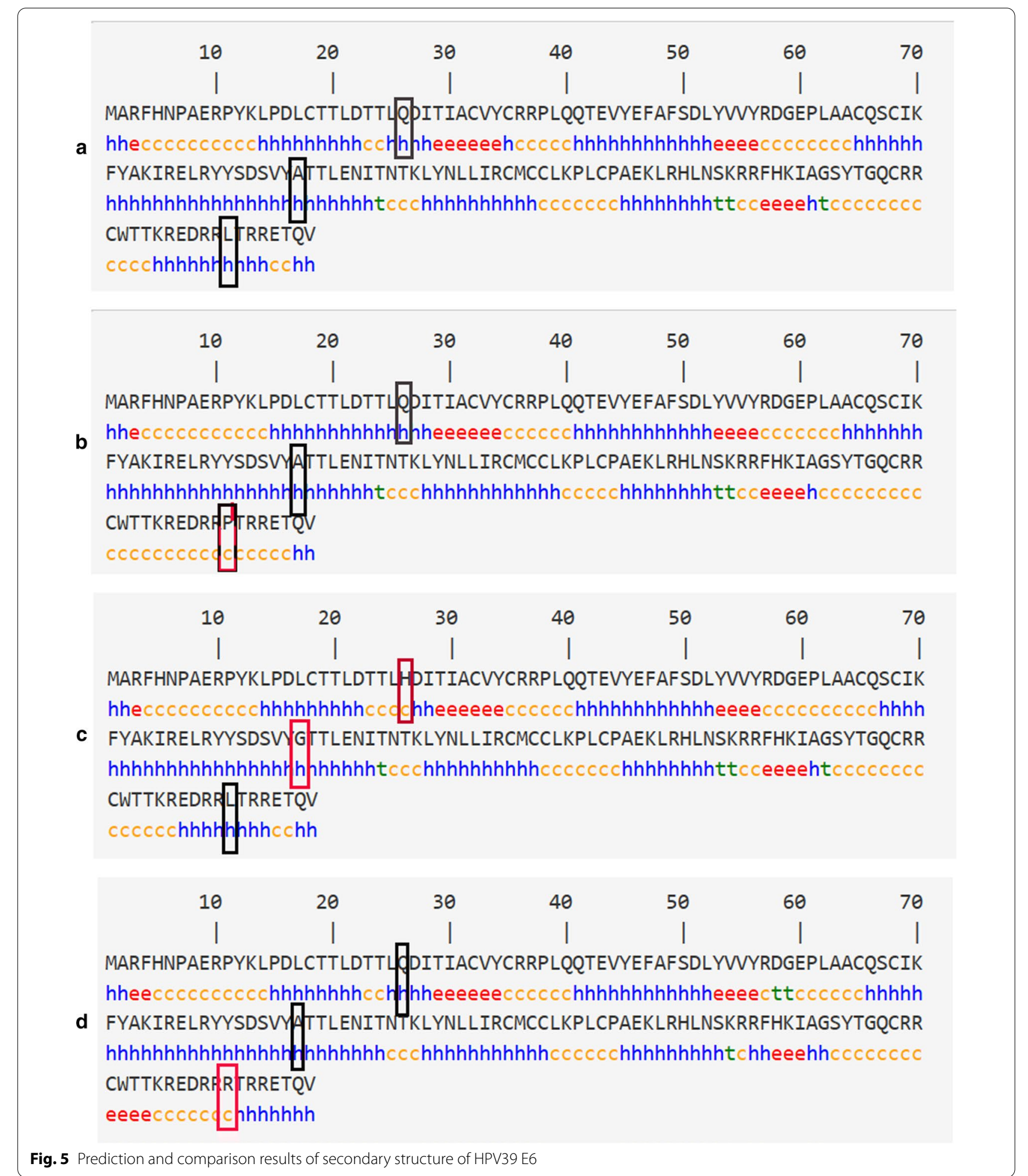

94.63\% for $L 1$ ) suggesting A pedigree should be emphasized in the development of HPV39 vaccine, HPV39 $\mathrm{B}$-pedigree is rare, and no B-pedigree mutant were reported previously in China. In this study, 39pe04 and 39pl04 two B-pedigree mutation sequences were isolated and pathogenicity of B-pedigree was stronger than that of A-pedigree, suggesting that more attention 
a

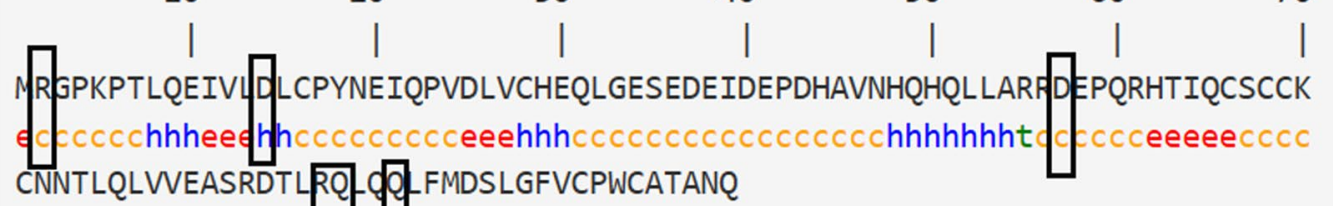
CNNTLQLVEASRDTLR요 FMDSLGFVCPWCATANQ

cctheeeeeecchhhh hh hh hhhhhhhhecccccccc

b
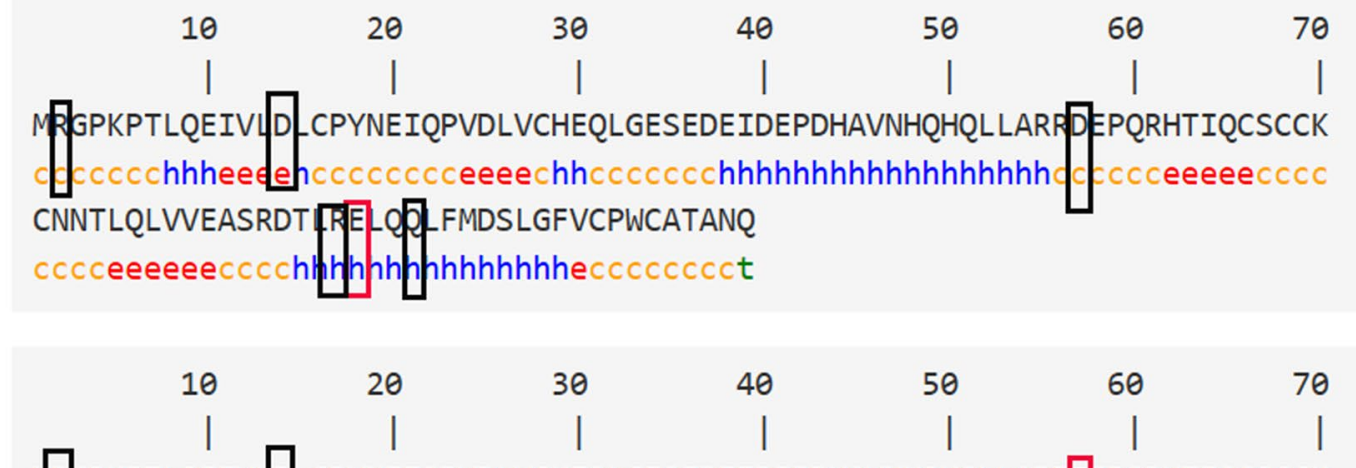

c

RSPKPTLQEIV D DCPYNEIQPVDLVCHEQLGESEDEIDEPDHAVNHQHQLLARREEPQRHTIQCSCCK CNNTLQLVVEASRDTLQR-ORLFMDSLGFVCPWCATANQ

ttcheeeeecchhhth hh hhhhhhhhhecccccccct
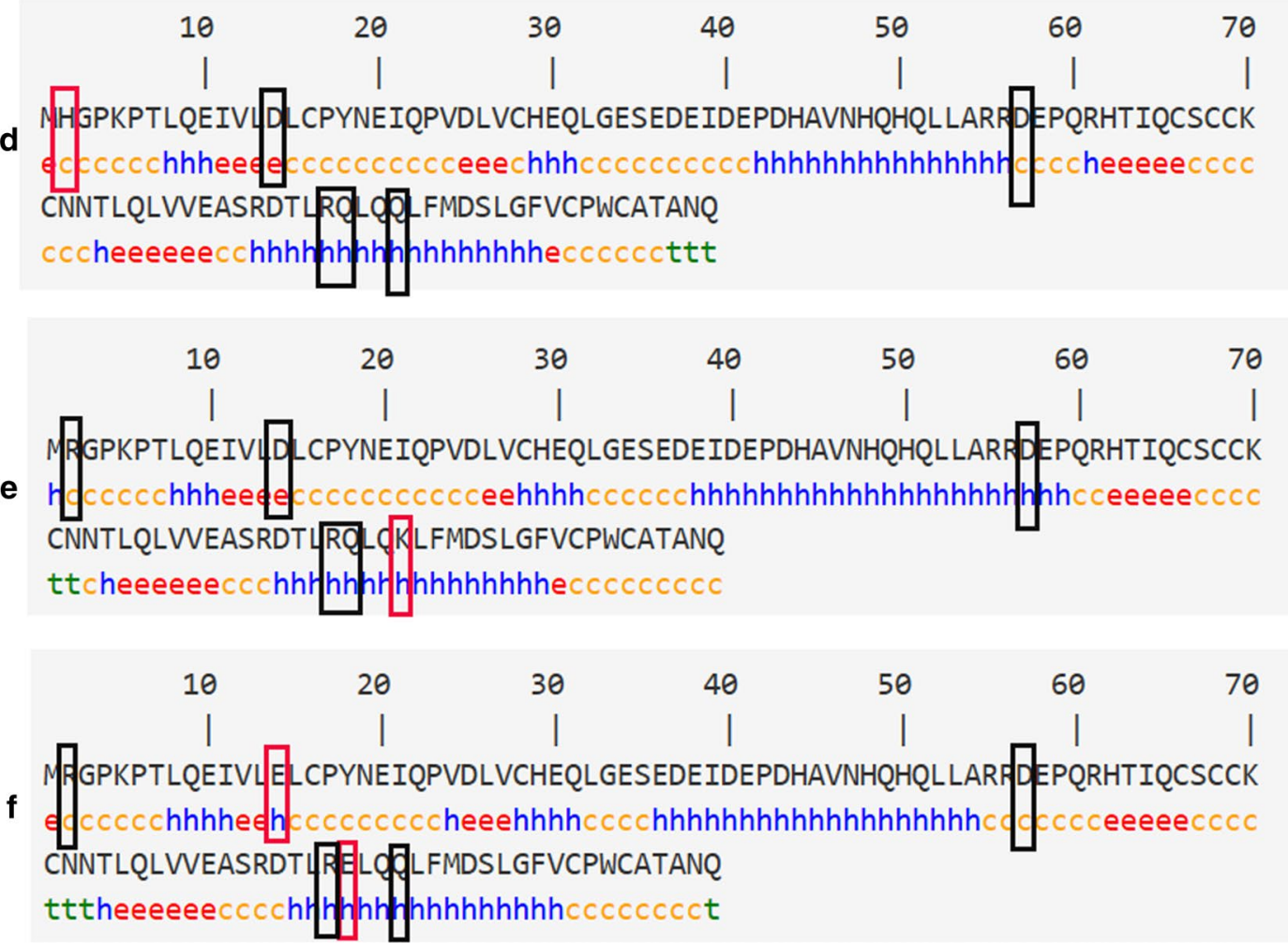

Fig. 6 Prediction and comparison results of secondary structure of HPV39E7 

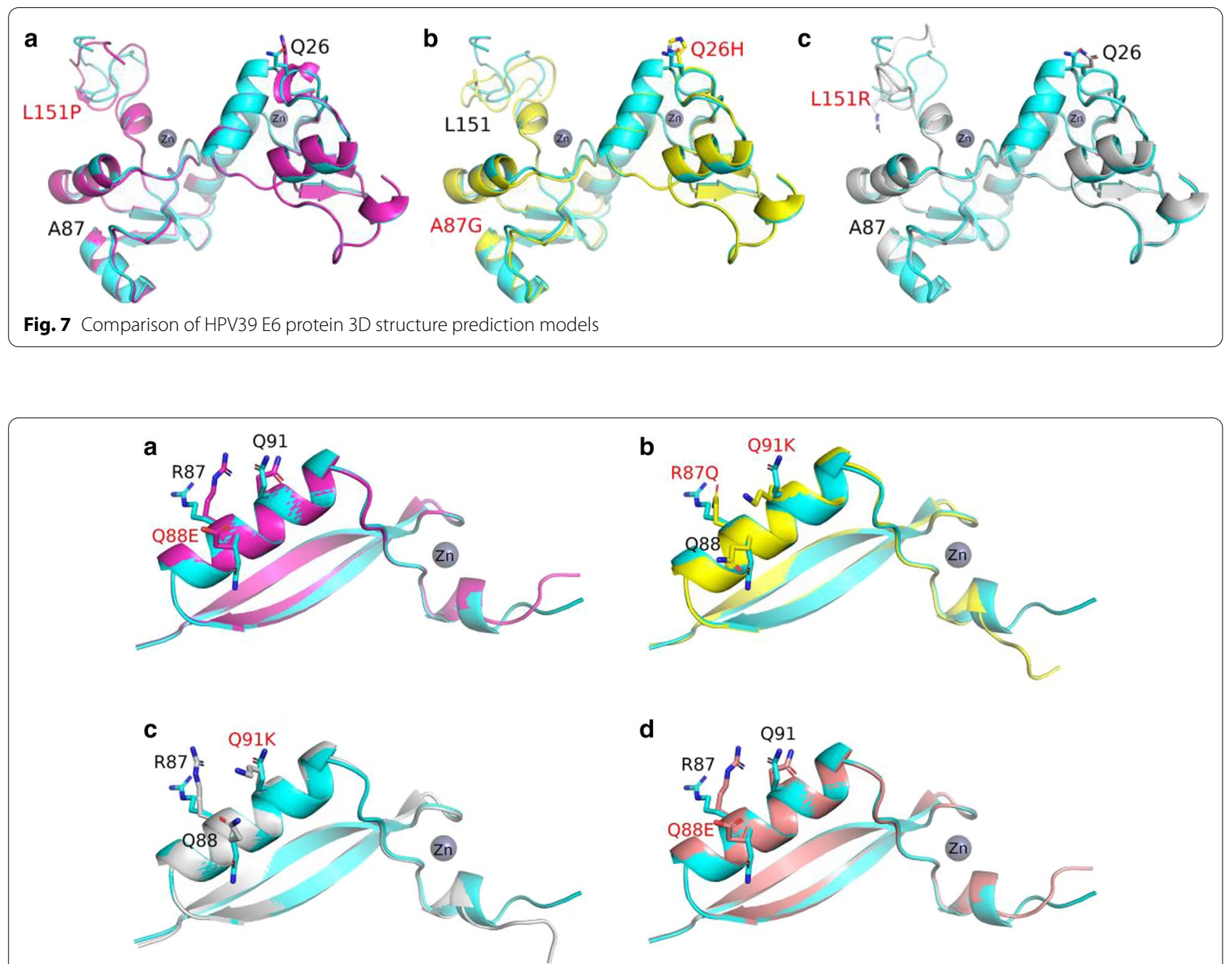

Fig. 8 Comparison of HPV39 E7 protein 3D structure prediction models

should be paid to the prevalence and prevention of B-pedigree HPV39 in the future.

Positive site selection may affect the virus infection efficiency and immunogenicity. The positive selection sites in $\mathrm{E} 6$ are 26, 87, 151, and in L1 are 75, 180, 222, 272, 284, 346, 356, no positive selection sites in E7 were detected, the positive selection sites may play an important role in improving the survival ability of HPV39 (e.g., resistance to immune response) and adapting to evolution. The codons 45, 138, 309 and 381 in L1 are negative selection sites, no mutation site was detected in this study, suggesting that mutation at these sites may harmful to the virus survival and evolution, so more attention should be pay to the mutation effect of these sites in the future research.

E6 and E7 are main carcinogenic protein and plays an important role in cervical lesions. Amino acid replacement may affect the carcinogenic potential of E6 and E7 protein and the biological functions of protein were mainly determined by its secondary and three-dimensional structure. The secondary structures of L151P (T558C), L151R (T558G) and Q26H (G596A) in E6 were changed from $\mathrm{H}$ to $\mathrm{C}$ by comparing with the reference sequence. These amino acid substitutions also have some effect on the secondary structures around them $(\mathrm{H}$ shortened and C increased). 145-149 were PDZ domaincontaining combined regional that was the target of E6 proteins for cellular transformation, crystal structures reveal that 152R-R-R-E-T-Q-V158 of E6 peptide are involved in PDZ recognition $[28,29]$. The regions in the half of carboxy terminal is mainly involved in the p53 binding [30], the L151P, L151R of HPV39 located in the surfaces of HPV E6 and close to the PDZ binding, recognition region that probability influence the function of P53 binding and degradation. E6 has four Cys-X-XCys repetitions, respectively located amino acid residues 32-35, 65-68, 105-108 and 138-141, Cys-X-X-Cys 
motifs have been suggested to coordinate metal binding and are frequently found in nucleic acid binding proteins [31]. A87G and Q26H close to the Zinc, those mutation may influence the protein binding and degradation. Meanwhile 26, 87, 151 are the positive selection sites in E6 and play an important role in improving the survival ability of HPV39, the positive selection sites screening results also verified the effects of the above mutations on protein function to some extent. For HPV39 E7, R2H and D57E appeared in C, D14E, R87Q, Q88E and Q91K appeared in $\mathrm{H}$, and there was no difference with M62849 secondary structure. HPV E7 protein was divided into three main domains $C R$ (conserved region)1, aa 1-15; CR2, aa 16-37; and CR3, aa 38-98, CR1 and CR2 mediate binding of viral oncoproteins to overlapping cellular proteins, including the product of retinoblastoma gene (pRB), cyclin A and cyclin E [32, 33]. 21-29 associated with the binding of retinoblastoma ( $\mathrm{Rb})$ tumor suppressor, since E7 acts as the major immortalizing protein through $\mathrm{Rb} / \mathrm{E} 2 \mathrm{~F}$ pathway, amino acid alternations in $\mathrm{Rb}$ binding domain may lead to the change of E7's ability to immortalize cells among variants [34]. The CR3 domain contains two "Cys-X-X-Cys" sequences located at amino acid residues 58-61 and 91-94 participating in $\mathrm{Zn}$ binding, are the most important regions associated with the transforming ability of E7 protein $[32,35]$. The zinc finger structure at the C-terminal was reported essential for HPV16 E7 to stabilize its structure and biological function, the disruption of the $91 \mathrm{Cys}-\mathrm{X}-\mathrm{X}-\mathrm{Cys} 94$ motif that lies towards the carboxyl terminus of E7 protein appeared to result in a greatly impaired transforming ability and reduced transactivation [32, 35]. The mutations in 91th amino-acid caused the loss of the zinc-binding CR3 domain of E7, resulting in a highly unstable protein that can be quickly degraded, and generating more epitopes to be presented in the MHCI pathway, so does the Q91H [36]. $\mathrm{R} 2 \mathrm{H}$ located in metal binding motifs, the transformation of E7 has been demonstrated to be eliminated by the mutation in 2th amino acid [35]. D14E located in CR1, mediate protein binding; D57E, R87Q, Q88E close to 58Cys-X-X-Cys61 and 91Cys-X-X-Cys94, that participating in $\mathrm{Zn}$ binding, the amnio acid substitution may influence the transforming ability of the E7 protein. These non-synonymous mutations slightly change the E6/E7 amino terminal and the trend of the carboxyl end structure disorder, A certain degree of secondary and threedimensional structure dislocation were existed between the reference and mutant sequence. Structure changes may lead to the different binding ability to the host p53, $\mathrm{RB}$ protein and other potential proteins, that may affect the pathogenicity of HPV39. Therefore, it is necessary to study the function of these non-synonymous mutations furtherly.
This study enrichment the data of HPV39 gene polymorphism and evolution in Chinese population, as well as provide a molecular biological basis for the future research analysis of HPV39 E6/E7/L1 and the design of therapeutic vaccine. However, due to the sample size limitation, the result of this study still has some shortcoming. Therefore, it is necessary to carry out multiregion and large sample population research, conduct a series of cell experiments to verify the above mutation sites function, explore the relationship between HPV39 gene polymorphism and genital lesions as well as its action mechanism.

\section{Conclusion}

HPV39 is a high prevalence HPV type in Southwest China, which is prone to mixed infection with other HPV types, especially with HPV6. $18-28$ years old is the highest HPV infection rate age group. A pedigree HPV39 E6, E7 and L1 gene is dominant in southwest china, two B-pedigree mutant sequences were isolated and its pathogenicity was stronger than A-pedigree. Positive selection sites may affect the virus infection efficiency, immunogenicity and improve the survival ability of HPV39 as well as adaptation to evolution. Negative selection sites suggesting that mutation at these sites may harmful to the virus survival and evolution. A certain degree of dislocation was existed between the protein secondary and three-dimensional structure of the reference and mutant sequence. The changes of structure may lead to the different binding ability to the host $\mathrm{p} 53$ protein and other potential proteins, thus affect the pathogenicity of HPV39.

\section{Abbreviations}

CC: Cervical cancer; CR: Conserved region; VLP: Virus-like particles; pRB: Retinoblastoma gene; HPV: Human papillomavirus; dsDNA: Double stranded DNA; MHC: Major histocompatibility complex; HR-HPV: High-risk human papilloma virus; MEGA 6: Molecular Evolutionary Genetics Analysis version 6.0.

\section{Acknowledgements \\ The authors would like to thank the following hospitals for the sample collection: Sichuan Reproductive Health Research Center Affiliated Hos- pital, Chengdu Song ziniao Sterility Hospital, Infertility Hospital Affiliated to Chengdu Medical College, Angel Women's and Children's Hospital, and Chengdu Jinsha hospital.}

\section{Authors' contributions}

$J H, X D, T L$ and YW conceived and designed the study; JH, XD, JD, XW, YC and QL collected the samples; SM, ZS and YL performed the experiments. JH and $X D$ wrote the manuscript, TL revised the manuscript. All authors read, edited, and approved the final manuscript for submission.

\section{Funding}

This work was funded by Key Scientific Research Foundation Projects of Sichuan Province (No. 2018JY0601). The fund is used for research design, data collection, analysis, interpretation, and manuscript writing. 


\section{Availability of data and materials}

All data generated or analyzed during this study are included in this article and GeneBank.

\section{Declarations}

\section{Ethics approval and consent to participate}

The present study was approved by the Education and Research Committee and the Ethics Committee of Sichuan University (Chengdu, China; approval number SCU20100196494). Before sample collection, a written informed consent was obtained from the patients or their guardians, and the privacy of patient/study was protected carefully.

\section{Consent to publication}

All patients participating in this study have consent for publication.

\section{Competing interests}

The authors declare that they have no competing interests.

\begin{abstract}
Author details
${ }^{1}$ Key Laboratory of Bio-Resources and Eco-Environment of Ministry of Education, College of Life Sciences, Sichuan University, Chengdu 610065, Sichuan, People's Republic of China. ${ }^{2}$ Bio-Resource Research and Utilization Joint Key Laboratory of Sichuan and Chongqing, Chongqing Nanchuan Biotechnology Research Institute, Chongqing, Sichuan, People's Republic of China. ${ }^{3}$ The People's Hospital of Pengzhou, Pengzhou, Sichuan, People's Republic of China. ${ }^{4}$ Institute of Medical Genetics, College of Life Sciences, Sichuan University, Chengdu 610064, China.
\end{abstract}

Received: 27 August 2020 Accepted: 5 March 2021

Published online: 08 April 2021

\section{References}

1. Harari A, Chen Z, Burk RD, Health P. HHS Public Access. 2015;1-18.

2. Hu SY, Zheng RS, Zhao FH, Zhang SW, Chen WQ, Qiao YL. Trend analysis of cervical cancer incidence and mortality rates in Chinese women during 1989-2008. Acta Acad Med Sin. 2014;36:119-25.

3. Bosch FX, Lorincz A, Muñoz N, Meijer CJLM, Shah KV. The causal relation between human papillomavirus and cervical cancer. J Clin Pathol. 2002;55:244-65.

4. Martinez-Zapien D, Ruiz FX, Poirson J, Mitschler A, Ramirez J, Forster A, et al. Structure of the E6/E6AP/p53 complex required for HPV-mediated degradation of p53. Nature. 2016;529:541-5. https://doi.org/10. 1038/nature16481.

5. Doorbar J, Quint W, Banks L, Bravo IG, Stoler M, Broker TR, et al. The biology and life-cycle of human papillomaviruses. Vaccine. 2012;30:F55-70. https://doi.org/10.1016/j.vaccine.2012.06.083.

6. Kozomara RJ, Brankovic-Magic MV, Jovic NR, Stosic SM, Magic ZM. Prognostic significance of TP53 mutations in oral squamous cell carcinoma with human papilloma virus infection. Int J Biol Markers. 2007;22:252-7.

7. Lizano M, Berumen J, Garcı A. HPV-related carcinogenesis: basic concepts. Viral Types and Variants. 2009;40:428-34.

8. Hu H, Zhao J, Yu W, Zhao J, Wang Z, Jin L, et al. Human papillomavirus DNA, HPV L1 capsid protein and p16 INK4a protein as markers to predict cervical lesion progression. Arch Gynecol Obstet. 2018. https:// doi.org/10.1007/s00404-018-4931-1.

9. Kocjan BJ, Bzhalava D, Forslund O, Dillner J, Poljak M. Molecular methods for identi fi cation and characterization of novel papillomaviruses. Clin Microbiol Infect. 2015;21:808-16. https://doi.org/10.1016/j.cmi. 2015.05.011.

10. Bernard HU, Burk RD, Chen Z, van Doorslaer K, Hausen H. Classification of papillomaviruses (PVs) based on 189 PV types and proposal of taxonomic amendments. Virology. 2010;401:70-9. https://doi.org/10. 1016/j.virol.2010.02.002.

11. De Villiers EM, Fauquet C, Broker TR, Bernard HU, Zur HH. Classification of papillomaviruses. Virology. 2004;324:17-27.
12. Félix A, Alemany L, Tous $S$, de Sanjosé S, Bosch FX. HPV distribution in cervical cancer in Portugal: a retrospective study from 1928 to 2005. Papillomavirus Res. 2016;2:41-5.

13. Dadar M, Chakraborty S, Dhama K, Prasad M. Advances in designing and developing vaccines, drugs and therapeutic approaches to counter human papilloma. Virus. 2018. https://doi.org/10.3389/fimmu.2018. 02478.

14. Zeng Z, Yang H, Li Z, He X, Griffith CC, Chen X. Prevalence and genotype distribution of HPV infection in China : analysis of 51, $345 \mathrm{HPV}$ genotyping results from China's Largest CAP certified laboratory. J Cancer. 2016;7:1037.

15. Li K, Yin R, Li Q, Wang D. Analysis of HPV distribution in patients with cervical precancerous lesions in Western China. Medicine. 2016. https://doi.org/10.1097/MD.0000000000007304.

16. Kosakovsky Pond SL, Frost SD. Not so different after all: a comparison of methods for detecting amino acid sites under selection. Mol Biol Evolut. 2005;22(5):1208-22.

17. Pond SLK, Frost SD. Datamonkey: rapid detection of selective pressure on individual sites of codon alignments. Bioinformatics. 2005;21(10):2531-3.

18. Geourjon C, Deléage G. Sopma: Significant improvements in protein secondary structure prediction by consensus prediction from multiple alignments. Bioinformatics. 1995;11:681-4.

19. Yang J, Yan R, Roy A, Xu D, Poisson J, Arbor A, et al. HHS Public Access. 2015;12:7-8.

20. Peitsch MC. SWISS-MODEL and the Swiss-PdbViewer : an environment for comparative protein modeling. 2016.

21. Lilkova E, Petkov P, Ilieva N, Litov L. Towards Molecular Modeling of the Impact of Heparin-Derived Oligosaccharides on hIFN- $\gamma$ Binding. 2015;030008

22. Zur Hausen H. Papillomaviruses and cancer: from basic studies to clinical application. Nat Rev Cancer. 2002;2(5):342-50.

23. Narisawa-Saito M, Kiyono T. Basic mechanisms of high-risk human papillomavirus-induced carcinogenesis: Roles of E6 and E7 proteins. Cancer Sci. 2007;98:1505-11.

24. Strati K, Lambert PF. Role of Rb-dependent and Rb-independent functions of papillomavirus E7 oncogene in head and neck cancer. Cancer Res. 2007:67:11585-93.

25. Buck CB, Day PM, Trus BL. The papillomavirus major capsid protein L1. Virology. 2013;445:169-74. https://doi.org/10.1016/j.virol.2013.05.038.

26. Longuet M, Beaudenon S, Orth G. Two novel genital human papillomavirus (HPV) types, HPV68 and HPV70, related to the potentially oncogenic HPV39. J Clin Microbiol. 1996;34:738-44.

27. Cui F, Zhang Z, Xu J, Ding X, Mu X, Wan Q, et al. Genetic variability and lineage phylogeny of human papillomavirus type 45 based on E6 and E7 genes in Southwest China. Virus Res. 2018;255:85-9. https://doi.org/ 10.1016/j.gene.2016.07.039.

28. Ainsworth J, Thomas M, Banks L, Coutlee F, Matlashewski G. Comparison of $\mathrm{p} 53$ and the PDZ domain containing protein MAGI-3 regulation by the $\mathrm{E} 6$ protein from high-risk human papillomaviruses. Virol J. 2008;5:1-9.

29. Zhang Y, Dasgupta J, Ma RZ, Banks L, Thomas M, Chen XS. Structures of a Human Papillomavirus (HPV) E6 Polypeptide Bound to MAGUK Proteins: Mechanisms of Targeting Tumor Suppressors by a High-Risk HPV Oncoprotein. J Virol. 2007;81:3618-26.

30. Thomas M, Glaunsinger B, Pim D, Javier R, Banks L. HPV E6 and MAGUK protein interactions: determination of the molecular basis for specific protein recognition and degradation. Oncogene. 2001;20:5431-9.

31. Barbosa MS, Lowy DR, Schiller JT. Papillomavirus polypeptides E6 and E7 are zinc-binding proteins. J Virol. 1989;63:1404-7.

32. Liu JH, Zhang YL, Zhu LQ, Xu YY, Zhao M, Wu XX. Human papillomavirus type 16 mutant $E 7$ protein induces oncogenic transformation via upregulation of cyclin A and cdc25A. Virol Sin. 2008;23:352-62.

33. Tommasino $M$. The human papillomavirus family and its role in carcinogenesis. Semin Cancer Biol. 2014;26:13-21. https://doi.org/10. 1016/j.semcancer.2013.11.002.

34. Liu M, He Z, Xi L, Li J, Liu F, Liu Y, et al. The distribution and common amino acid polymorphisms of human papillomavirus (HPV)-31 Variants in 2700 Women from Northern China. PLoS ONE. 2014;9:5-10. 
35. Li YL, Ma ZL, Zhao Y, Zang J. Immunization with mutant HPV16 E7 protein inhibits the growth of TC-1 cells in Tumor-Bearing mice. Oncol Lett. 2015;9:1851-6.

36. Robles-Rodríguez OA, Pérez-Trujillo JJ, Barrón-Cantú JA, Torres-Cerda A, Gutiérrez-Puente Y, García-García A, et al. Antitumor effect of adenoviruses expressing mutant non-oncogenic E7 versions from HPV-16 fused to calreticulin. J BUON. 2020;25:543-8.

\section{Publisher's Note}

Springer Nature remains neutral with regard to jurisdictional claims in published maps and institutional affiliations.
Ready to submit your research? Choose BMC and benefit from:

- fast, convenient online submission

- thorough peer review by experienced researchers in your field

- rapid publication on acceptance

- support for research data, including large and complex data types

- gold Open Access which fosters wider collaboration and increased citations

- maximum visibility for your research: over $100 \mathrm{M}$ website views per year

At BMC, research is always in progress.

Learn more biomedcentral.com/submissions 